\title{
Synaptic Self-Organization of Spatio-Temporal Pattern Selectivity
}

\author{
Mohammad Dehghani Habibabadi ${ }^{*}$ and Klaus Richard Pawelzik ${ }^{1}$ \\ ${ }^{1}$ Institute for Theoretical Physics, University of Bremen, 28359 Bremen, Germany \\ *mohammad@neuro.uni-bremen.de
}

\section{ABSTRACT}

Spiking model neurons can be set up to respond selectively to specific spatio-temporal spike patterns by optimization of their input weights. It is unknown, however, if existing synaptic plasticity mechanisms can achieve this temporal mode of neuronal coding and computation. Here it is shown that changes of synaptic efficacies which tend to balance excitatory and inhibitory synaptic inputs can make neurons sensitive to particular input spike patterns. Simulations demonstrate that a combination of Hebbian mechanisms, hetero-synaptic plasticity and synaptic scaling is sufficient for self-organizing sensitivity for spatio-temporal spike patterns that repeat in the input. In networks inclusion of hetero-synaptic plasticity leads to specialization and faithful representation of pattern sequences by a group of target neurons. Pattern detection is found to be robust against a range of distortions and noise. Furthermore, the resulting balance of excitatory and inhibitory inputs protects the memory for a specific pattern from being overwritten during ongoing learning when the pattern is not present. These results not only provide an explanation for experimental observations of balanced excitation and inhibition in cortex but also promote the plausibility of precise temporal coding in the brain.

\section{Author summary}

Neurons communicate using action potentials, that are pulses localized in time. There is evidence that the exact timing of these so called spikes carries information. The hypothesis, however, that computations in brains are indeed based on precise patterns of spikes is debated particularly because this would require the existence of suitable detectors. While theoretically individual neurons can perform spike pattern detection when their input synapses are carefully adjusted it is not known if existing synaptic plasticity mechanisms support this coding principle. Here, a combination of basic but realistic mechanis ms is demonstrated to self-organize the synaptic input efficacies such that individual neurons become detectors of patterns repeating in the input. The proposed combination of learning mechanisms yields a balance of excitation and inhibition similar to observations in cortex, robustness of detection against perturbations and noise, and persistence of memory against ongoing plasticity without the learned patterns. It enables groups of neurons to incrementally learn sets of patterns thereby faithfully representing their 'which' and 'when' in sequences. These results suggest that computations based on spatio-temporal spike patterns might emerge without any supervision from the synaptic plasticity mechanisms present in the brain.

\section{Introduction}

Despite decades of research, it is still debated which coding schemes are used in central nervous systems. While in early sensory areas of cortex, stimuli appear to be represented mostly by spike rates, it cannot 
be disputed that temporal information is faithfully processed. While this can in principle be achieved by modulated spike rates in large populations of neurons it is tempting to hypothesize that at least in higher areas, as i.e., frontal cortex, temporally precise responses of individual neurons play an important role. In fact, experimental studies on visual, auditory, olfactory, and somato-sensory cortex indicate that neurons can respond rather deterministically to inputs, underlining the possibility of precise spike codes. ${ }^{1-8}$.

A range of theoretical studies attempted to elucidate mechanisms that could support precise coding of spatio-temporal patterns ${ }^{9,10}$. It was found that with suitable synaptic weights, even simple integrate-andfire neurons are sensitive to specific spatio-temporal input spike patterns. The Tempotron ${ }^{9}$ was introduced as an extension of the Perceptron ${ }^{11}$. It performs classification of spatio-temporal patterns with a spike response to patterns only from a given set with a supervised algorithm for potentiating and depressing a neuron's afferents. The number of patterns that a neuron can learn to classify depends on their length and the time constants of the neurons and the synaptic inputs ${ }^{12}$. While in the Tempotron the action potential is allowed to occur anywhere during the time of the learned patterns, it was later shown that neurons can be forced to fire also at a specific time ${ }^{10}$ during a specific pattern's presence, which can be achieved by several more or less realistic synaptic mechanisms ${ }^{13,14}$. Both the Tempotron and the Chronotron employ supervised learning mechanisms based on label and time, respectively.

Supervised learning of spatio-temporal patterns seem at odds with reality, where the input is not labeled, impinges on the neuron continuously, and is subject to distortions and noise. In particular, they would need to explain how synaptic plasticity mechanisms become informed which aspect of the data should be taken into account when a label comes only long after the patterns. A recent study addressed this latter problem. It showed that neurons can recognize spatio-temporal patterns embedded in a background of noise using only weak supervision where the known number of repetitions of a pattern is used for optimizing synaptic efficacy ${ }^{15}$. Based on the N-methyl-D-aspartate (NMDA) receptor, a learning rule was proposed ${ }^{15}$ that yields similar results as obtained by optimization. The biological plausibility of this correlation-based rule, however, is questionable. It does not respect Dale's rule since synapses can change their sign, it is still supervised, and it requires a careful selection of potential weight changes such that only a given small percentage of potential changes become effective.

Therefore, it remains an open question if existing mechanisms of synaptic plasticity can be identified which enable neurons to specialize on statistically dominant patterns in the temporal stream of their inputs neurons in an entirely unsupervised manner.

The starting point of our considerations is synaptic scaling: It has been shown that neurons do not remain silent for long periods, but scale their weights to achieve a genetically intended number of spikes 16. Then, we use basic Hebbian mechanisms for the plasticity of both, excitatory and inhibitory neurons which for excitatory synapses resemble the NMDA-receptor. Dale's law is enforced, i.e. inhibitory and excitatory neurons can not change into one another throughout the learning process. Furthermore, upper bounds on synaptic efficacies are imposed which prohibit the well-known runaway instability of Hebbian mechanisms. For excitatory synapses, we finally employ hetero-synaptic plasticity mechanisms that provide negative changes of efficacy, induce synaptic competition and thereby serve specialization ${ }^{17}$.

A combination of these realistic mechanisms turn out to be sufficient for self-organization of pattern detection in single neurons. At times when no pattern is present excitatory and inhibitory inputs become globally balanced, leading to high specificity of detection. During the time when a learned pattern is present we observe detailed balance, where excitatory and inhibitory inputs cancel each other in temporal detail. These results parallel findings of global and detailed balance of excitation and inhibition in cortex 
$18-20$.

The resulting synaptic efficacies are then shown to ensure robust pattern recognition that is resistant to temporal jitter and noise. When basing learning on jittered patterns and also on Poisson rate modulations instead of precisely repeating patterns we obtain even more robust pattern detection. These results underline that the proposed mechanisms can be based on imprecise patterns and temporally modulated rate codes.

Next, we wondered if and how learned memory traces vanish during ongoing plasticity when only random patterns are presented which contain no statistically dominant structures. We find extremely long memory persistence already for a single output neuron. This leads to the question if the proposed mechanisms might contribute to solving the stability-plasticity problem ${ }^{21}$, such that they would support incremental learning where patterns occur rarely and are intermingled with random activity and/or different patterns. We investigated this for groups of output neurons where competition for patterns is induced by pre-synaptic hetero-synaptic plasticity ${ }^{22}$. Thereby the output neurons specialize on different subsets of patterns such that the group as a whole self-organizes faithful representation of the 'which' and 'when' of patterns in the input. The memory persistence in this setting is finally shown to allow for incremental learning of sets of patterns in neuronal populations.

\section{Results}

In all simulations we consider simple leaky integrate and fire neurons with a fixed membrane time constant of $15 \mathrm{~ms}$ and pre-synaptic spikes originating from 500 input neurons ( $80 \%$ excitatory and 20\% inhibitory). They provide input currents via kernels that have the shape of alpha-functions. For each synapse, the amplitudes of the input currents depend on a single parameter, the synaptic weight. The kernels have different time constants for excitatory and inhibitory synapses. The signs of the weights are not allowed to change during learning, i.e., Dale's law is enforced.

\section{Single post-synaptic neuron}

Synaptic plasticity is based on correlations between the input-kernels and deflections of the membrane potentials with respect to a threshold. For changes of the inhibitory synapses, positive deflections increase the weights, and negative deflections lead to their decay. For changes of the excitatory synapses, we let only positive deflections contribute, mimicking NMDA-dependent mechanisms. Without further constraints, this latter Hebbian mechanism is unstable.

Runaway instabilities are avoided by a combination of three simple but biologically highly plausible mechanisms: First, unbounded growth is made impossible by clipping the weights at upper limits for excitatory and inhibitory synapses. Second, positive weight changes for excitatory synapses are quenched when the long time activity exceeds a pre-determined rate, which mimics synaptic scaling ${ }^{16}$. Then no positive weight changes can take place and only the signals for decreasing excitatory weights come into effect. Third, negative weight changes for excitatory synapses are induced by hetero-synaptic plasticity. Specifically, we include post-synaptic hetero-synaptic plasticity where the potential weight changes of different afferents are made dependent such that the resources needed for strong increases of the postsynaptic contributions to synaptic efficacies are taken from synapses which would otherwise increase only weakly. Thereby the latter synapses' efficacies become reduced; however, this does not lead to the normalization of excitatory weights (see Materials and methods).

It turns out that these ingredients are sufficient for robust self-organization of spatio-temporal spike pattern detection in single neurons. As an example, Fig 1 shows the membrane potential (MP) of a single neuron before and after learning a random pattern of length $50 \mathrm{~ms}$ that has the same statistics as the 
random background but repeats in every training epoch of length $2000 \mathrm{~ms}$.

(a)

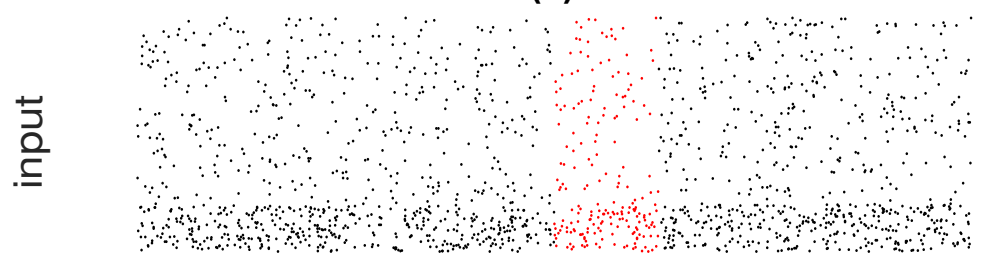

(b)

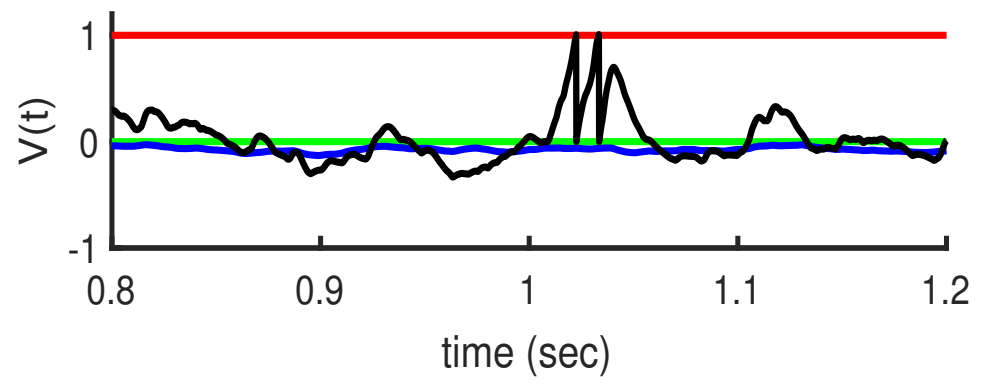

Fig 1. a: Input activity in the raster plot, five hundred afferents ( $80 \%$ excitatory and $20 \%$ inhibitory) send inputs to one post-synaptic neuron. The excitatory neurons' rate is $5 \mathrm{~Hz}$, and the inhibitory neurons' rates are $20 \mathrm{~Hz}$. There is a random embedded pattern of length $50 \mathrm{~ms}$ in the red area. b: Membrane potential versus time. The black trace shows that after learning fluctuations outside of the embedded pattern are attenuated, and two spikes occurring the embedded pattern. The green line is for resting potential, red is for threshold, blue is for before learning, and black is for after learning.

After convergence of the weights we separated the excitatory and inhibitory inputs to see how their respective contributions lead to firing only during the pattern (Fig 2 a).

Fig 2 a indicates that inhibitory and excitatory inputs cancel each other outside of the embedded pattern in the mean (global balance). Fig $2 \mathrm{~b}$ depicts the inhibitory and excitatory parts from filterning the inputs by the membrane dynamics which add to the MP (here we also include the reset mechanism). Averaging on epochs (Fig 2 c) explicitly shows that global balance removes fluctuations in the mean. In contrast, during the time of the embedded pattern, the respective contributions to the membrane potential both increase but remain mostly balanced also across time (detailed balance). In fact, only some residual imbalance between excitatory and inhibitory afferents allow the post-synaptic neuron to fire during this period of time. The detailed balance during the embedded pattern is reflected by a substantial anti-correlation between excitatory and inhibitory afferents at zero time shift ( Fig $2 \mathrm{~d}$ ) .

To quantify the performance of the learning mechanism for ensembles of random patterns, we consider the average percentage $R$ of spikes that correctly detect the pattern:

$$
R=\left\langle\frac{n_{s}^{p}(L)}{n_{s}+\varepsilon}\right\rangle_{\mu}
$$

where $n_{s}$ is the total number of spikes during a testing period and $n_{s}^{p}$ the spikes' number related to the presence of the pattern to be detected. Since patterns can induce spikes also shortly after the pattern 
(a)

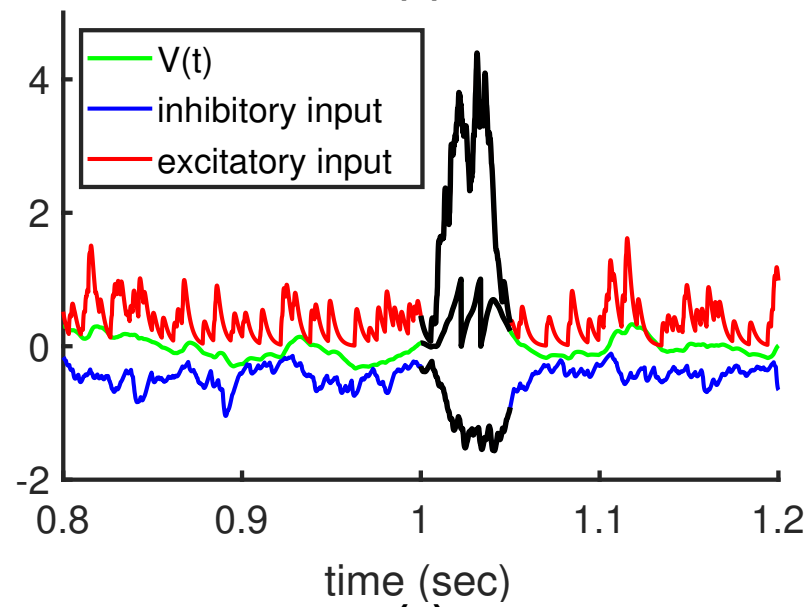

(c)

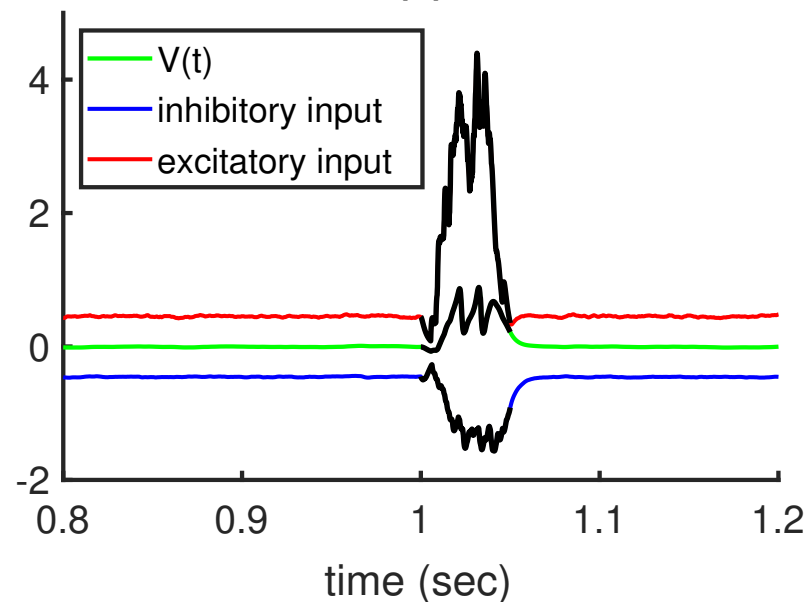

(b)

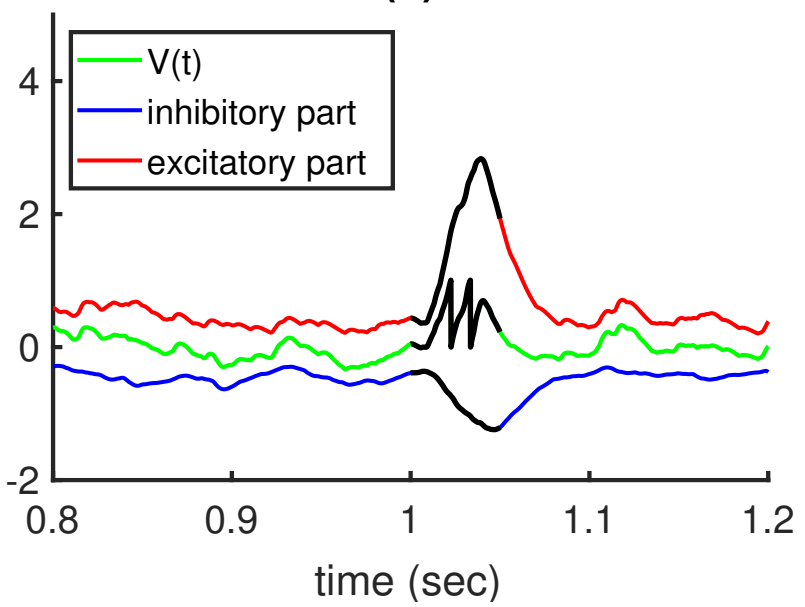

(d)

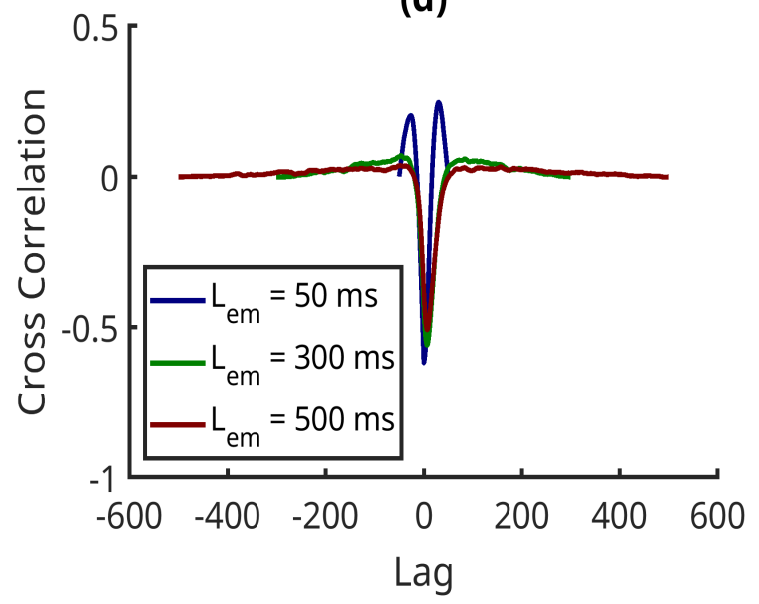

Fig 2. a,b,c: A fixed pattern is embedded between $1000 \mathrm{~ms}$ and $1050 \mathrm{~ms}$, i.e. duration $L_{e m}=50 \mathrm{~ms}$. a: excitatory and inhibitory inputs and the membrane potential after convergence of self-organization with this pattern. $b$ : contributions of excitation and inhibition to the MP from filtering the respective inputs by the neurons's membrane potential dynamics. c: Average contribution of excitatory and inhibitory inputs from 1000 epochs with different epochs. d: cross-correlation between inhibitory and excitatory inputs for neurons that where exposed to patterns of $L_{e m}=50,300,500 \mathrm{~ms}$ (shown in their afferents starting from $1000 \mathrm{~ms}$.). The shown correlations shown are averages from 1000 simulations with random patterns. In all figures, the length of the training epoch is $2000 \mathrm{~ms}$, and the desired number of spikes is 2, i.e., the desired firing rate is $r_{0}=1 \mathrm{~Hz}$.

due to the finite decay time of the excitatory synaptic kernel, the time window for testing if spikes occur inside the pattern is extended by $L$ ms after the pattern has ended. Adding an arbitrary small number to the denominator ensures a definite result $(R=0)$ when no spikes occur at all. The ratio is averaged for an ensemble of independent embedded patterns, $\mu$.

Fig 3a shows that neurons learn to fire only in response to the embedded pattern and remain silent otherwise. Extending the testing window by $L=15 \mathrm{~ms}$ reveals that the performance becomes perfect. Fig $3 \mathrm{~b}$ shows that using longer embedded patterns allows neurons to learn them faster: a more extended embedded pattern has more space for residual excitatory-inhibitory imbalances and therefore can more rapidly find the embedded pattern. 
(a)

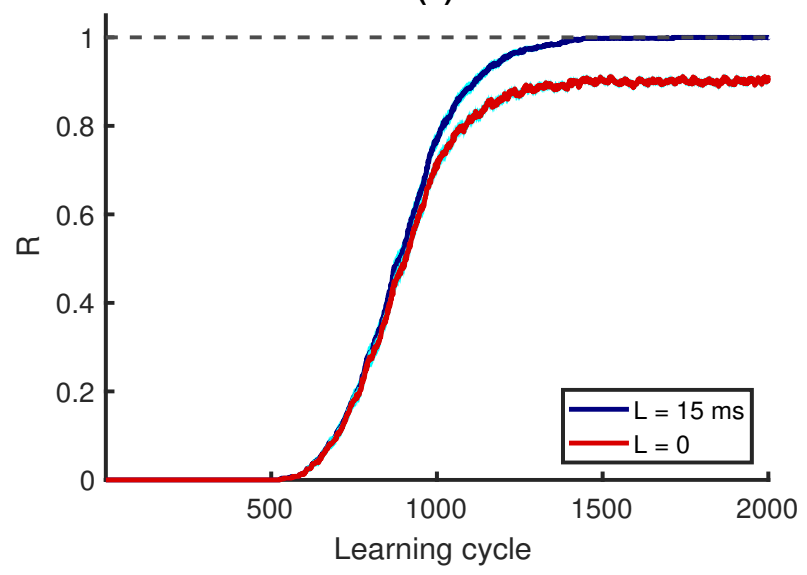

(b)

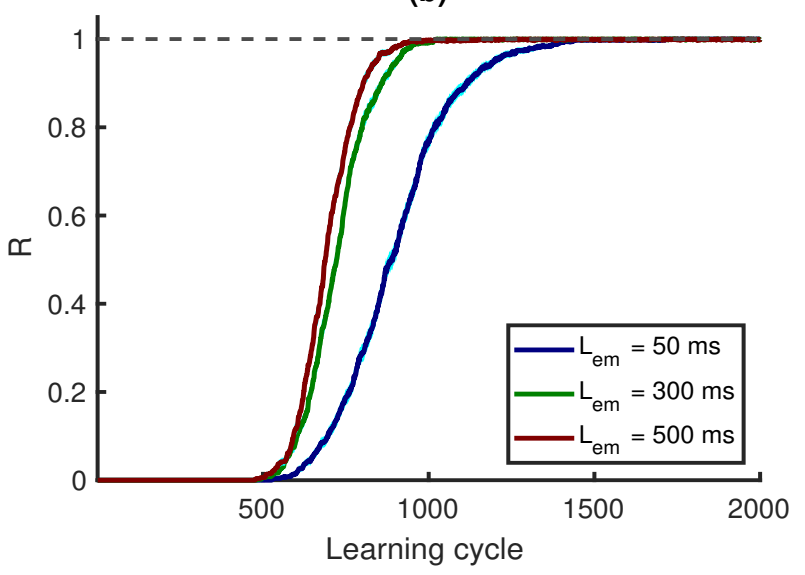

Fig 3. (Color online) a: Learning performance $\mathrm{R}$ versus learning cycle, for $L=0$ and $L=15 \mathrm{~ms}$. A 50 ms pattern is embedded between 1000 and $1050 \mathrm{~ms}$. b: $\mathrm{R}$ versus learning cycle, $L=15 \mathrm{~ms}$. Patterns duration, $L_{e m}$, are 50,300, and $500 \mathrm{~ms}$, shown in afferents from $1000 \mathrm{~ms}$. $\mathrm{R}$ is an average of 500 simulations, in which there are 500 afferents, and the length of the training epoch is $2000 \mathrm{~ms}$. The desired firing rate is $r_{0}=1 \mathrm{~Hz}$.

\section{Noise and memory robustness}

We wondered if and how the memory for the originally learned pattern decays when synaptic plasticity is present during long periods of random inputs where an already learned pattern does not re-appear. Note, plasticity was switched off when we tested whether it remembers the originally learned pattern. Fig 4 a shows that even after 20000 learning cycles, 80 percent of spikes would still occur during the embedded pattern time, (chance level is 0.025 ). This striking memory persistence can be understood by considering that due to the synaptic scaling inherent in the learning mechanism, the neurons will change synapses until the pre-determined long-term firing rate is achieved also for random patterns (red in Fig 4 b). Since the inputs have no structure the weights mostly become only scaled, which does not erase the selectivity for the learned pattern. When the learned pattern is then again presented to the afferents, the neuron fires additional spikes during the pattern, which leads to a much higher firing rate for the pattern (green in Fig 4 b).

The plausibility of spike pattern coding depends on its robustness against noise and pattern distortions. For neurons that have learned a particular pattern without noise we examined the dependency of detection performance on three types of noises:

First: we removed spikes inside the embedded patterns and examined if neurons can still recognize the embedded pattern. Fig 5a shows the robustness against removing spikes inside the embedded pattern.

Second: during learning, the neuron receives inhibitory input at a frequency of $20 \mathrm{~Hz}$ and excitatory input at a frequency of $5 \mathrm{~Hz}$ (ratio 4 to 1 ). For testing we added $S$ random spikes per second to excitatory input neurons and $4 \times S$ spikes per second into inhibitory neurons. When excitatory neurons have $S$ spikes extra in their input, the new rate becomes $r_{e}^{\text {new }}=r_{e}+S$. Fig $5 \mathrm{~b}$ shows that the performance $\mathrm{R}$ decays when $r_{e}^{\text {new }}>10 \mathrm{~Hz}$.

Third: in this part, we examine the robustness of detection against jitter noise. For this purpose we shuffle the times of the spikes in the afferents using a Gaussian distribution with zero mean and $\sigma$ standard deviation. The Fig $5 \mathrm{c}$ shows that the algorithm is robust until $\sigma=5 \mathrm{~ms}$, and after that, performance starts to decay. 

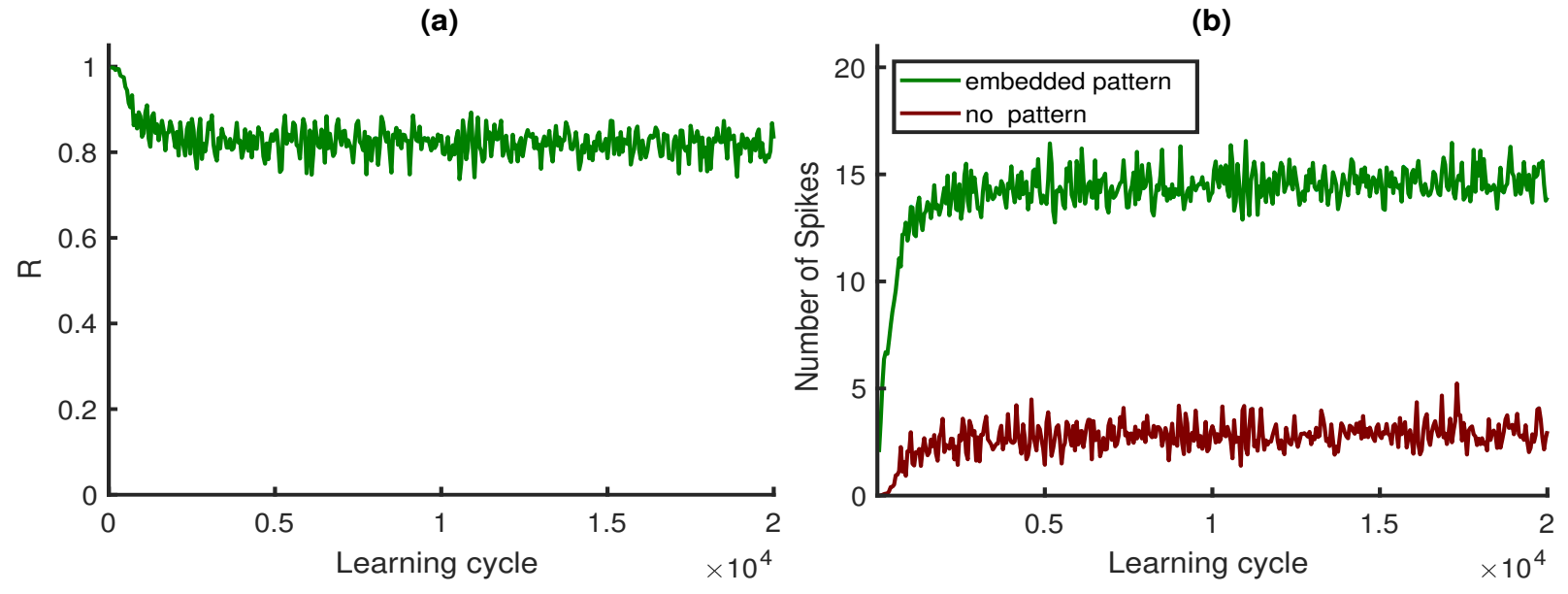

Fig 4. (Color online) a: $R$ as memory criterion versus learning cycle: Without learned patterns in afferents, learning continues, and every 50 cycles, learning is paused, and the learned pattern is placed in the background, then $\mathrm{R}$ is computed as a memory criterion. b: number of spikes versus learning cycle. Neuron elicits more spikes when the embedded pattern is in the afferents (green) than the situation without a pattern(red). Patterns duration is $50 \mathrm{~ms}$, shown in afferents starting from $1000 \mathrm{~ms}$, and this figure is based on an average of 500 simulations.

Next, we wondered if precise patterns are required for self-organizing pattern selectivity. First, we perturbed the training patterns by jittering the spikes according to a Gaussian distribution with mean zero and a standard deviation of $20 \mathrm{~ms}$. We found that this does not hamper learning. Fig 6a shows the R-value in each learning cycle; the blue line is for testing with the original pattern, the green line with the jittered patterns. For the red line, we dropped contributions to $\mathrm{R}$ from the epochs where no spikes at all occur. Then, we transformed the inputs to Poisson spike rates (see Materials and methods for details), leading to a similar result (Fig 6b, blue line is for testing with the original pattern, the green line for testing with Poisson spike rates including no spikes, the red when epochs with no spikes are dropped). It turns out, that with the corresponding weights pattern detection becomes more robust with respect to jitter noise (Fig 6c, the blue and red lines are for testing with different $\sigma$ for jittered and Poisson spike rates, respectively). These results demonstrate that codes based on rate modulations and spatio-temporal pattern detection by individual neurons are compatible.

\section{Diversification by pre-synaptic hetero-synaptic plasticity}

In the approach presented so far a neuron can become a detector for more than only one pattern, along the lines of the Tempotron ${ }^{9}$ (Fig 7 ). Its activity would then, however, obscure which individual pattern was present at which time. For a faithful representation of a sequence of patterns, as e.g. the phonemes in spoken human language, it is therefore desirable that different neurons in a network become specialized for different subsets of patterns such that as a whole a network represents precisely the which and when of patterns in a sequence. If successful, such a system could e.g. be used for unsupervised speech recognition 15 .

As a first step into this direction we wondered which realistic synaptic mechanism might enforce specialization of different neurons in a network for different pattern sets. We found that already synaptic competition induced by pre-synaptic hetero-synaptic plasticity ${ }^{22}$ yields sufficient selectivity for different patterns in an ensemble of neurons such that identity and order of patterns are represented faithfully (for 
(a)

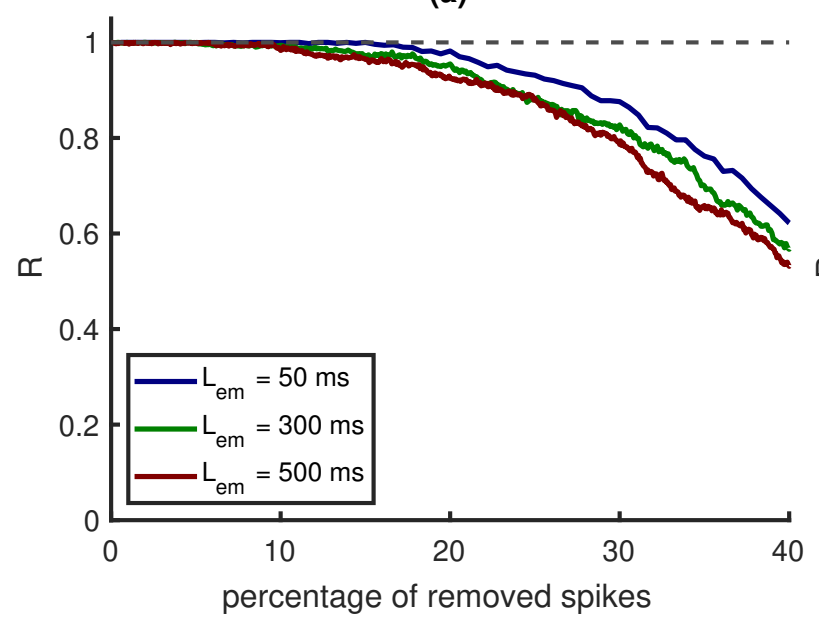

(b)

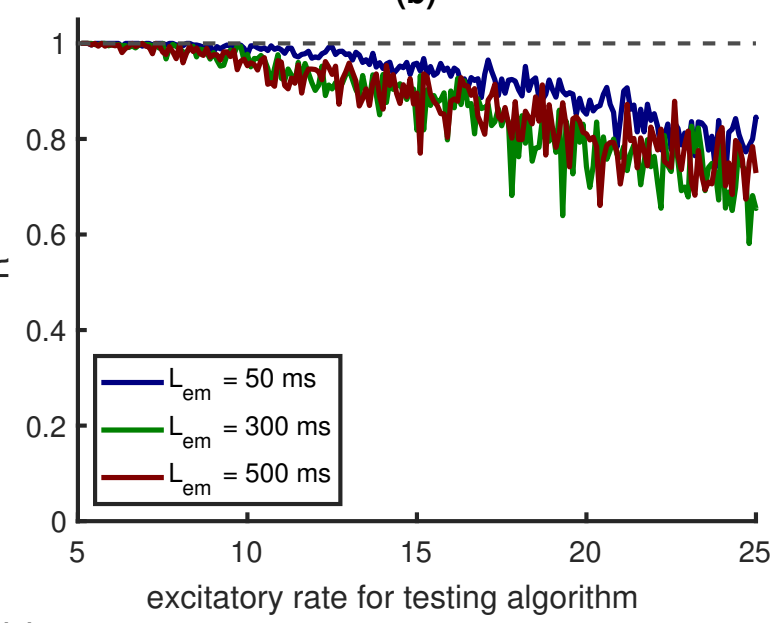

(c)

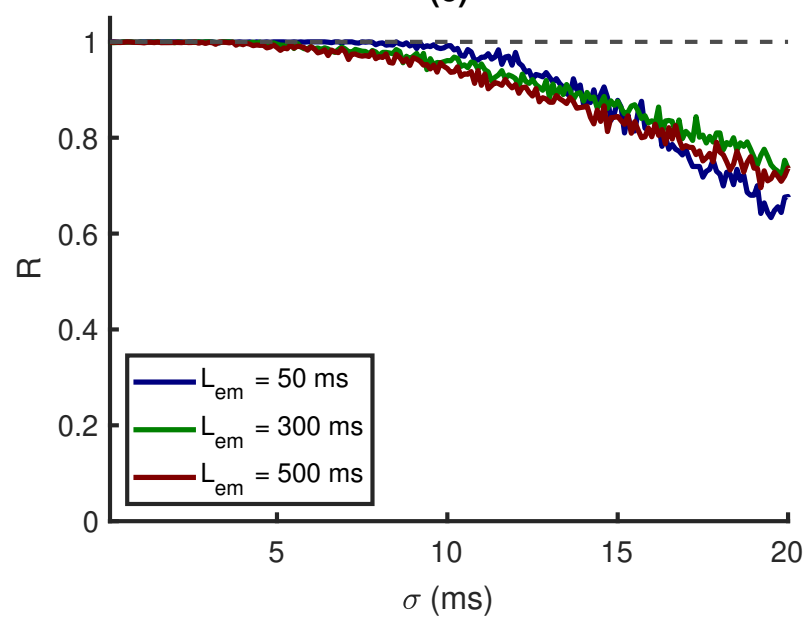

Fig 5. Effect of noise on the learning algorithm. a: removing spikes. b: increasing firing rates. c: Jitter noise. Patterns durations are 50, 300, and $500 \mathrm{~ms}$, shown in afferents starting from 1000 milliseconds. This figure is based on an average of 500 simulations, in which there are 500 afferents, the length of the training epoch is $2000 \mathrm{~ms}$, and $r_{0}=1 \mathrm{~Hz}$.

details of the computational implementation see Materials and methods).

To quantify the performance, we use the rank of a matrix where each row represents one of the neurons, and each column one of the patterns. The matrix element in each row is set to 1 if the correspondingly neuron is active for that pattern, and to 0 otherwise. The rank of this matrix provides the number of linearly independent row vectors. When it meets the number of the different patterns in a given stimulus the population of neurons faithfully represents the presence and the order of the patterns. Therefore, we introduce the ratio of the rank of this matrix to the number of patterns as performance measure $\Omega$.

As an example, we trained networks of different sizes with four patterns where always all patterns were present in each training epoch. We found that the pre-synaptic competition leads to selectivity for all four patterns as soon as 14 post-synaptic neurons were present. In contrast, without this pre-synaptic competition, separation does not become complete.(Fig 8 a).

We then tested the persistence of learned selectivities (with 14 neurons) when learning is continued with random input. We tested performance (with plasticity switched off) and found practically no decay of $\Omega$ even when learning was continued 10X longer than it takes for learning all patterns. 
(a)

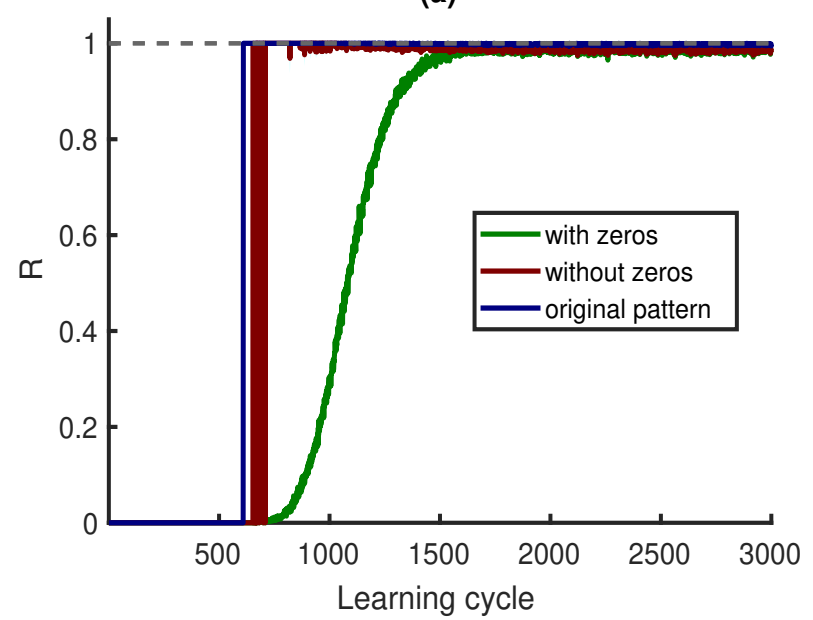

(b)

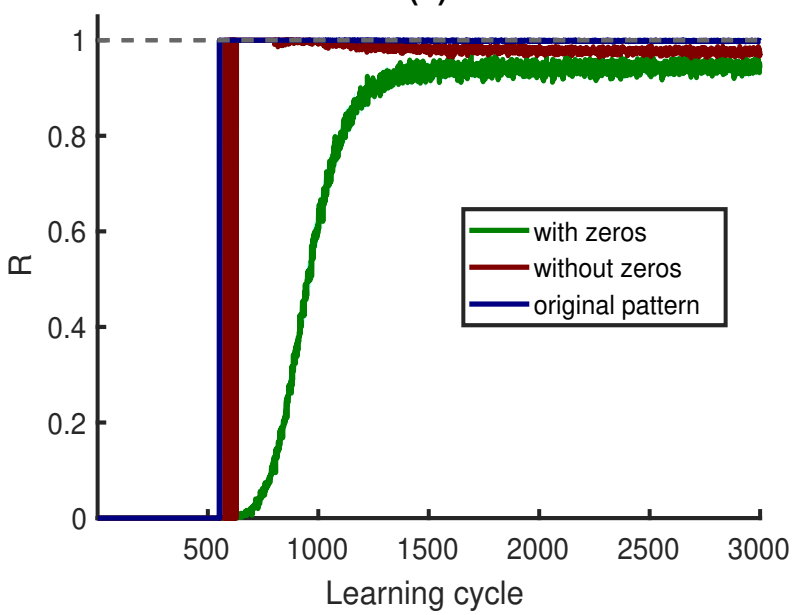

(c)

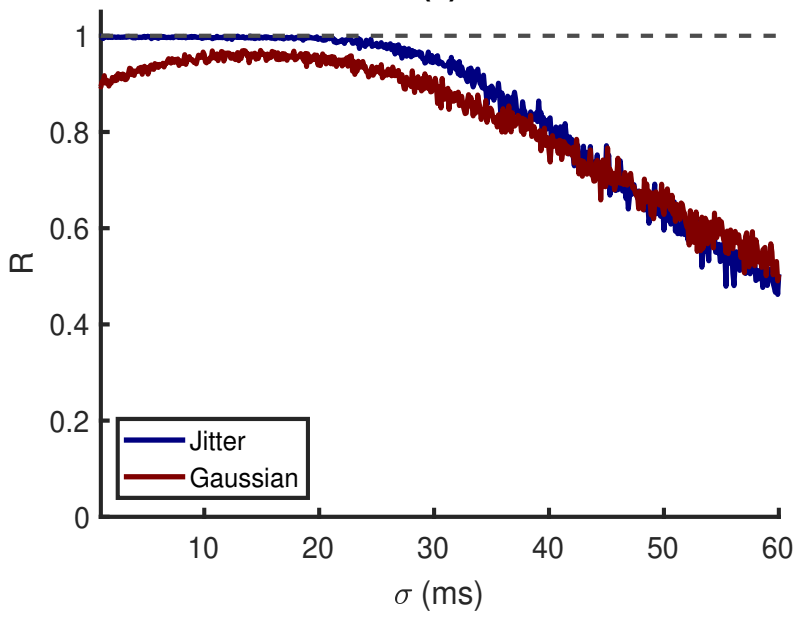

Fig 6. Learning with jittered patterns and Poisson spike rates: a: the training patterns are perturbed by jittering the spikes with a Gaussian distribution with a standard deviation of $\sigma=20 \mathrm{~ms}$ and a zero mean. Blue represents testing with the original pattern, the green line represents jittered data with no spikes in MP, and the red line represents dropping episodes with no spikes in MP. b: Poisson spike rate learning: the blue line represents testing with the original pattern, the green line represents testing with Poisson spike rates, including those with no MP spikes, and the red line shows $\mathrm{R}$ when dropping episodes with no spikes. c: the blue and red lines represent tests of jittered and Poisson spike rates, respectively, with varying $\sigma$. This figure is based on an average of 500 simulations with 500 afferents. The training epoch is $2000 \mathrm{~ms}, L_{e m}=50 \mathrm{~ms}$, and $r_{0}=1 \mathrm{~Hz}$.

This result motivated us to test if learning a set of patterns is possible from learning epochs that contain only subsets of all patterns. Our preliminary investigations indicate that such incremental learning is indeed possible. As an example, we considered 4 embedded patterns and 14 post-synaptic neurons. Each embedded pattern has a 0.2 probability of being present in each learning cycle in the epoch. Note that thereby some learning cycles have no pattern in the epoch. Also, we randomly chose locations from $\left(\left[300300+L_{e m}\right),\left[400400+L_{e m}\right), \ldots\left[17001700+L_{e m}\right] \mathrm{ms}\right)$ to put the embedded patterns in them. As Fig $8 \mathrm{~b}$ shows the relative rank $\Omega$ goes to one, indicating that patterns can be learned also incrementally. 
(a)

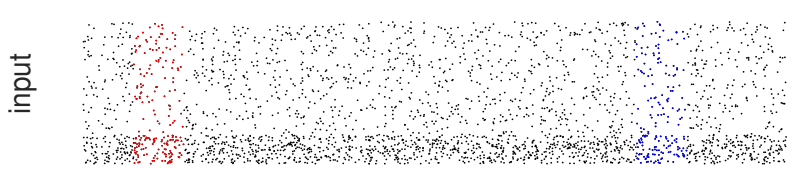

(b)

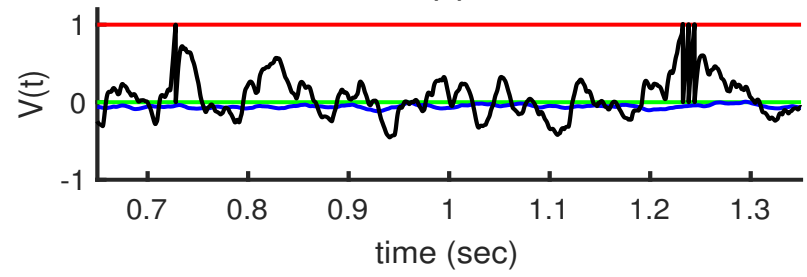

Fig 7. Example of membrane potential versus time after learning with two patterns: a: Input activity in the raster plot, five hundred afferents (80\% excitatory and 20\% inhibitory) send inputs to one post-synaptic neuron. The excitatory neurons' rate is $5 \mathrm{~Hz}$, and the inhibitory neurons' rates are $20 \mathrm{~Hz}$. There are two random embedded patterns of length $50 \mathrm{~ms}$ in the red and blue areas, $r_{0}=2 \mathrm{~Hz}$, and the length of the training epoch is $2000 \mathrm{~ms}$. b: Membrane potential versus time. The black trace shows that after learning neuron responds two both embedded patterns. The green line is for resting potential, red is for threshold, blue is for before learning, and black is for after learning.

(a)

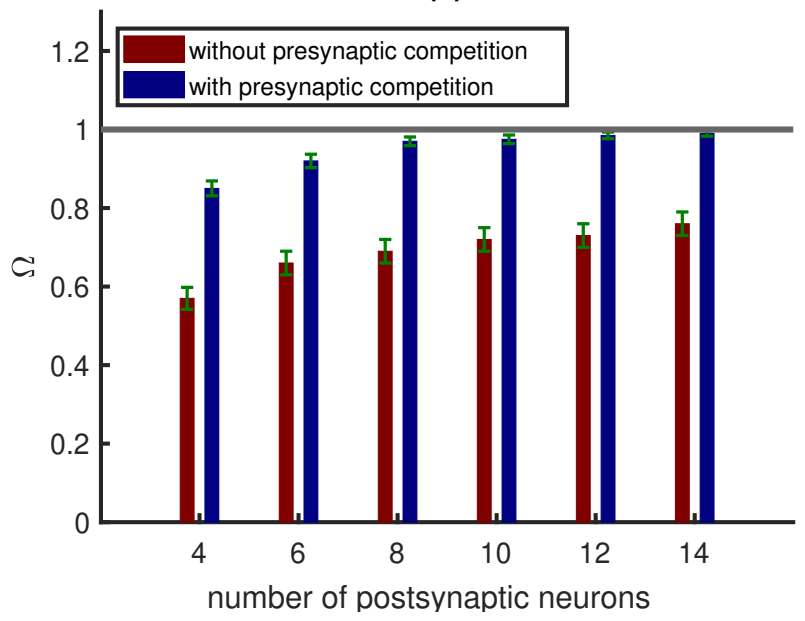

(b)

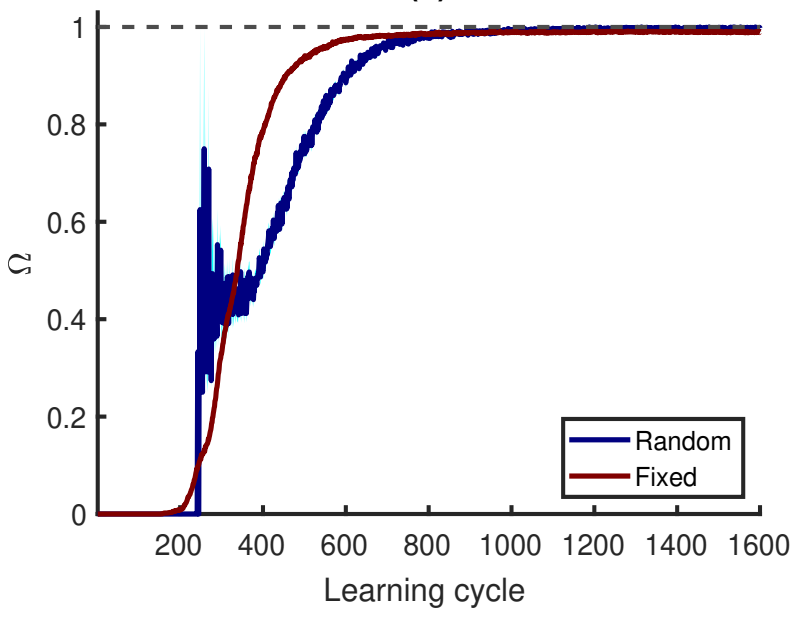

Fig 8. In all simulations, there are 14 post-synaptic neurons, 4 independent embedded patterns, with duration $50 \mathrm{~ms}$, and $r_{0}=1 \mathrm{~Hz}$. a: The system's $\Omega$ (rank/4) versus the number of post-synaptic neurons after learning. b: $\Omega$ versus learning cycle. red line: Each pattern is shown in each learning cycle in a fixed position. blue line: Each pattern is shown in the epoch with the probability of 0.2 at a random place. $a$ is based on an average of 50 simulations, and $b$ is based on an average of 500 simulations.

\section{Discussion}

Neurons respond faithfully to input sequences ${ }^{23}$. That is, they are rather deterministic devices and with suitable synaptic efficacies, individual neurons can, in principle, serve as detectors for specific spatiotemporal input spike patterns. This opens the possibility that coding and computation in brains is at least in part based on temporally precise action potentials. 
In the past, this hypothesis has been investigated in simple integrate and fire models. Supervised learning rules were proposed that enable neurons to signal the presence of a pattern ${ }^{9}$ and to fire at predefined time points during a specific pattern ${ }^{10}$. It was further demonstrated that relatively weak supervision can be sufficient for learning the synaptic weights for pattern detection ${ }^{15}$. Here, only the number of pattern occurrences is needed for the specialization of a neuron ${ }^{15}$. While a learning rule for this 'aggregate label learning' was rigorously derived, the proposed biological realization suffers from several rather unrealistic assumptions. In particular, excitatory and inhibitory plasticity are not treated separately, and Dale's law was not observed. When the signs of synapses may change, this has also a negative impact on the excitatory and inhibitory balance. For some patterns, there may be only few inhibitory neurons remaining in the system after learning. When there is a lack of inhibition; however, the potential can take a value close to the threshold, increasing the chance of getting random spikes outside the embedded patterns. Also, a selection criterion was used, by which independently of their sign, only the largest $10 \%$ of changes were taken into account, for which no realistic interpretation was provided.

Therefore, it remains an open question if the synaptic plasticity mechanisms present in real neuronal networks support a coding scheme that is based on spati-temporal patterns of spikes.

We found that a combination of Hebbian mechanisms, hetero-synaptic competition, and synaptic scaling indeed makes individual neurons sensitive for statistically dominant spatio-temporal patterns in their afferents. Performance is found to be robust to temporal jitter, missing spikes, and additional noise. In particular, also patterns consisting of Poisson spike rate modulations are captured surprisingly well by the proposed plasticity mechanisms and lead to robust detection (Fig 6 b, c ).

The proposed combination of learning mechanisms yields a detailed balance of excitation and inhibition where this is possible: during the learned pattern. This fits nicely to experimental observations 18 revealing a negative correlation between excitatory and inhibitory inputs. Outside the pattern, global balance is achieved ${ }^{20}$.

Balance is a natural consequence of Hebbian mechanisms when they are simultaneously present in both excitatory and inhibitory synapses, and the otherwise unstable growth of excitatory efficacies is constrained. While this has been noted before ${ }^{24}$, we here show that Hebbian plasticity can select synaptic efficacies that make neurons detectors for spatio-temporal patterns when realistic constraints are taken into account. In particular, we found that a subtle interplay of the instability of Hebbian mechanisms for excitatory synapses and synaptic scaling enforces a local imbalance during the learned patterns which leads to specific and temporally precise spike responses.

It has been shown that the inhibitory neurons also stabilize responses ${ }^{25}$, and that excitatory-inhibitory balance protects memory for rate patterns in neural networks 24,26 . Our simulations now demonstrate that also the memory for spike patterns is protected from being overwritten by noise or another patterns.

After successful learning of patterns, the random background input does not lead to spikes in individual neurons. When then plasticity is continuously present for a long time during which only random inputs are present, the input weights mainly become scaled up until the desired number of spikes is reached, i.e. the plasticity mechanisms considered here are consistent with synaptic scaling ${ }^{16}$. Obviously, scaling preserves the memory for the learned patterns, which will then induce far more spikes than the background. In other words, the plasticity mechanisms discussed here lead to a very long memory persistence already in a single neuron such that selectivity is preserved and sensitivity becomes even enhanced. We tested the memory persistence also in groups of neurons that specialized for subsets of the input patterns via pre-synaptic hetero-synaptic plasticity. Here, we observed practically no decay. This finding suggests that in groups of neurons incremental learning of sets of patterns should be possible, where in each successive 
training epoch only a subset or even none of all patterns is present. We could confirm this hypothesis for simple cases; however, the question if the combination of plasticity mechanisms discussed here indeed provides a solution for the stability-plasticity dilemma ${ }^{21}$ in spatio-temporal pattern learning will require more systematic investigations which go beyond the scope of this paper.

The fact that biologically realistic plasticity mechanisms can support the self-organization of spatiotemporal pattern detection by individual neurons underlines the possibility that such temporal codes are indeed present in nervous systems. Particularly the ability to learn patterns underlying Poisson spike rates demonstrates that such a temporal coding scheme can be consistent with rate codes. We speculate that this transformation of temporally modulated rates to spike pattern codes could explain the increase in sparsity observed in early visual cortex when natural contexts are included ${ }^{27}$ as well as the extreme sparsity of activations in higher cortical areas.

\section{Materials and methods}

\section{Neuron model}

In this work, we employ the leaky integrate and fire (LIF) model. The dynamic of the membrane potential of a single neuron $V(t)$ which is receiving current from $N$ afferents is:

$$
\tau_{m} \frac{d}{d t} V(t)=-V(t)+R_{m} I_{\text {external }}
$$

where $\tau_{m}$ and $R_{m}$ are time constant and resistance of the membrane, respectively. We consider $R=1$. Whenever the neuron arrives or passes the threshold it evokes spike output and resets to resting potential. Resting potential is zero in this study, and $I_{\text {external }}$ is the current from excitatory (E) and inhibitory (I) afferents, which is

$$
I_{j}=\sum_{i=1}^{N_{E}} w_{i j}^{E} \sum_{t_{i}^{j}<t} K^{E}\left(t-t_{i}^{j}\right)+\sum_{i=1}^{N_{I}} w_{i j}^{I} \sum_{t_{i}^{j}<t} K^{I}\left(t-t_{i}^{j}\right)
$$

where $w_{i j}^{E, I}$ represents the respective ith afferent's excitatory and inhibitory synaptic strength to output neuron $j . N_{E}$ and $N_{I}$ are the numbers of excitatory and inhibitory afferents, respectively $\left(N=N_{E}+N_{I}\right)$. Note that at time $t_{i}^{j}$, there is a spike in afferent $i$, and at this time, afferent $i$ send input to the post-synaptic neuron(s) as much as $w_{i} \times K$. The shape of the kernel is an alpha-function with the following equation:

$$
K\left(t-t_{i}^{j}\right)=A\left[\exp \left(-\frac{t-t_{i}^{j}}{\tau_{r}}\right)-\exp \left(-\frac{t-t_{i}^{j}}{\tau_{d}}\right)\right]
$$

where $\tau_{r}$ and $\tau_{d}$ are time constants of synaptic current, which are different for excitatory and inhibitory synapses. In this study $\tau_{m}=15 \mathrm{~ms}$. $\mathrm{A}$ is normalisation factor and it is $\frac{\eta^{\eta / \eta-1}}{\eta-1}$, where $\eta=\frac{\tau_{r}}{\tau_{d}}$. To numerically integrate equation 2 , we use Euler method with $\Delta t=0.1 \mathrm{~ms}$. The parameters and learning rates are written in Table 1.

\section{Learning algorithm}

We assume that a synapse's efficacy is the product of pre- and post-synaptic components

$$
w_{i j}=a_{i j} b_{i j}
$$




\begin{tabular}{|l|l|l|}
\hline Symbol & Description & Value \\
\hline \hline$\tau_{m}$ & membrane time constant & $15 \mathrm{msec}$ \\
\hline$\tau_{r}^{e}$ & Rise time of Excitatory neurons & $3 \mathrm{msec}$ \\
\hline$\tau_{r}^{i}$ & Rise time of Inhibitory neurons & $5 \mathrm{msec}$ \\
\hline$\tau_{d}^{e}$ & Decay time of Excitatory neurons & $0.5 \mathrm{msec}$ \\
\hline$\tau_{d}^{i}$ & Decay time of Inhibitory neurons & $1 \mathrm{msec}$ \\
\hline$r_{e}$ & Rate of external input to Excitatory neurons & $5 \mathrm{~Hz}$ \\
\hline$r_{i}$ & Rate of external input to Inhibitory neurons & $20 \mathrm{~Hz}$ \\
\hline $\mathrm{N}$ & Number of neurons & 500 \\
\hline$N^{E}$ & Number of Excitatory neurons & 400 \\
\hline$N^{I}$ & Number of Inhibitory neurons & 100 \\
\hline$\lambda$ & Inhibitory learning rate & $10^{-4}$ \\
\hline$\rho$ & Excitatory learning rate (one post-synaptic neuron) & $0.5 \times 10^{-4}$ \\
\hline$\gamma$ & Excitatory learning rate (more than one post-synaptic neuron) & $0.8 \times 10^{-4}$ \\
\hline $\mathrm{g}$ & acceleration constant & 20 \\
\hline$\Delta t$ & time step & $0.1 \mathrm{~ms}$ \\
\hline
\end{tabular}

Tab 1. List of parameters.

where $a_{i j}$ is provided from the pre-synaptic neuron $i$, and $b_{i j}$ is provided by the post-synaptic neuron $j$. If we have $N$ pre-synaptic neurons $(i=1, \ldots, N)$ and $M$ post-synaptic neurons $(j=1, \ldots, M)$ small changes $\delta a_{i j}$ and $\delta b_{i j}$ lead to

$$
\delta w_{i j}=a_{i j} \delta b_{i j}+\delta a_{i j} b_{i j}+\delta a_{i j} \delta b_{i j}
$$

We assume that correlations between pre-synaptic input and post-synaptic membrane potential drive weight changes. By considering only the spike at time $t_{i}^{l}$ the correlation between $i^{\prime} t h$ afferent and the $j^{\prime} t h$ post-synaptic neuron's potential $V_{j}(t)$ is:

$$
\vartheta_{i j}^{l}=\int_{0}^{\infty} d t k\left(t-t_{i}^{l}\right)\left[V_{j}(t)-V_{0}\right]
$$

where $V_{0}$ is an arbitrary threshold that is set to zero in all simulations of this study. In every learning epoch all $n_{i}$ spikes in the $i^{\prime} t h$ afferent are used for updating the weights. As a result, we compute correlation for all spikes $l$ and add them together to determine the eligibility

$$
\varepsilon_{i j}=\sum_{l=1}^{n_{i}} \vartheta_{i j}^{l}
$$

which is the basic signal for weights changes at synapse $(i, j)$.

For inhibitory synapses weight changes are set to be simply proportional to $\varepsilon$.

For excitatory synapses changes are assumed to depend only on the positive part $\left[\varepsilon_{i j}\right]_{+}$which mimics the characteristics of the NMDA receptor . For excitatory input we also take synaptic scaling into account. That is, neurons tend to fire and to implement a specific firing rate $r_{0}$ that is genetically determined ${ }^{16}$. Therefore, if a post-synaptic neuron's long term firing rate $r_{j}$ is less than the desired $r_{0}$, it scales its afferent 
excitatory weights up. When the firing rate $r_{j}$ is larger than $r_{0}$, it does not provide resources for further growth. We implement this with a quantity $q_{j}$ :

$$
q_{j}=\left[\tanh \left(r_{0}-r_{j}\right)\right]_{+}
$$

Changes of synapses are subject to limitations of material provided by the respective pre and postsynaptic neurons (e.g., release sites, vesicles, receptor densities). Plausibly, this leads to competition for changes of different synapses, which affects the respective pre- and post-synaptic components a and b differently. In the current approach we include pre- and post-synaptic competition only for the changes of excitatory weights thereby modeling pre- and post-synaptic versions of hetero-synaptic plasticity $17,22,28,29$

\section{Single post-synaptic neuron}

For a single post-synaptic neuron $(j=1)$, we have $b_{i 1}=: b_{i}, \varepsilon_{i 1}=: \varepsilon_{i}, q_{1}=: q$, and $a_{i 1}=: a_{i}$.

Here inhibitory synapses are changed by

$$
\delta b_{i}^{-}=\lambda \varepsilon_{i}
$$

where $\lambda$ is the learning rate for inhibitory synapses. For simplicity we here set $a_{i}=1$ and $\delta a_{i j}=0$ (for the full version see next section).

For excitatory synapses we subtract the mean of the plasticity signals $\left[\varepsilon_{i}\right]_{+}$, to mimick post-synaptic hetero-synaptic plasticity:

$$
\tilde{\varepsilon}_{i}=\left[\varepsilon_{i}\right]_{+}-\frac{1}{\sum_{i=1} \theta\left(\varepsilon_{i}\right)} \sum_{i=1}^{N}\left[\varepsilon_{i}\right]_{+}
$$

where $\theta$ is the Heaviside step function. Note, this does not imply strikt normalization since Dale's law prevents negative changes that otherwise would turn excitatory synapses to inhibitory synapses.

The excitatory afferents weights then change according to the following equation:

$$
\delta b_{i}^{+}=\rho\left(q\left[\tilde{\varepsilon}_{i}\right]_{+}+\left[\tilde{\varepsilon}_{i}\right]_{-}\right)
$$

where $\rho$ is the learning rate.

\section{More than one post-synaptic neuron}

When there is more than one post-synaptic neuron each afferent has a different eligibility for each post-synaptic neuron (Eq. 8). Therefore $\delta b_{i j}$ needs to be taken into account (Eq.6).

For inhibitory synapses we use:

$$
\begin{aligned}
& \delta b_{i j}^{-}=\lambda \varepsilon_{i j} \\
& \delta a_{i j}^{-}=\lambda \varepsilon_{i j}
\end{aligned}
$$

As for single target neurons $j$ the changes of excitatory synapses are subject to post-synaptic heterosynaptic plasticity that we realize by subtracting the mean of eligibilities:

$$
\tilde{\varepsilon}_{i j}=\left[\varepsilon_{i j}\right]_{+}-\frac{1}{\sum_{i=1} \theta\left(\varepsilon_{i j}\right)} \sum_{i=1}^{N}\left[\varepsilon_{i j}\right]_{+}
$$

With this the post-synaptic components change by 


$$
\delta b_{i j}^{+}=\gamma\left(q_{j}\left[\tilde{\varepsilon}_{i j}\right]_{+}+\left[\tilde{\varepsilon}_{i j}\right]_{-}\right)
$$

where $\gamma$ is the learning rate. We assume that also pre-synaptic neurons have finite resources for increasing their contributions to synaptic efficacies $\left(a_{i j}\right)$, as e.g., release sites or vesicle densities. Fig 9 illustrates this pre- and post-synaptic competition. We restrict the competition to excitatory neurons that up-regulate synapses because this requires resources, whereas decreases of efficacies may even release resources. To apply the competition, we first identify the signals that would scale up excitatory synapses:

$$
C_{i j}=q_{j}\left[\tilde{\varepsilon}_{i j}\right]_{+} \cdot
$$

The pre-synaptic competition then is implemented by subtraction of the mean of $C_{i j}$

$$
B_{i j}=C_{i j}-\frac{1}{M} \sum_{j=1}^{M} C_{i j}
$$

where $\mathrm{M}$ is the number of post-synaptic neurons. Therefore

$$
\delta a_{i j}^{+}=\gamma\left(g B_{i j}+\left[\tilde{\varepsilon}_{i j}\right]_{-}\right)
$$

where $g$ is a constant to accelerate pre-synaptic competitions.

\section{Simulation details}

In all simulations, there are 500 afferents (80\% excitatory and $20 \%$ inhibitory), and the learning epoch length is $2000 \mathrm{~ms}$. Afferent and embedded pattern spikes are generated randomly with Poisson point processes in which the excitatory rate is $5 \mathrm{~Hz}\left(r_{e}\right)$, and the inhibitory rate is $20 \mathrm{~Hz}\left(r_{i}\right)$. Except for Fig 8 (blue line) all embedded patterns are in the epoch in every learning cycle.

In the network model, the initial efficacies of $a_{i j}$ and $b_{i j}$ synapses are chosen from a Gaussian distribution with a mean of $10^{-1}$ and standard deviations of $10^{-1}$. In the single post-synaptic neuron model, they are chosen from a Gaussian distribution with a mean of $10^{-2}$ and standard deviations of $10^{-3}$. Here we cut negative numbers.

Synaptic scaling depends on firing rates averaged over long times. Since we perform on-line learning we use a low pass filter such that the rate $r_{j}(t+1)$ of post-synaptic neuron $j$ used for learning in episode $t+1$ is a running average of the actual rates $\hat{r}_{j}$ in previous epochs:

$$
r_{j}(t+1)=0.1 r_{j}(t)+0.9 \hat{r}_{j}(t)
$$

Furthermore, plasticity is not instantaneous but depends on an accumulation of signals for weight changes over some time. We implement this also by a low pass filter such that the changes taking place in each epoch $\delta a_{i j}(t+1), \delta b_{i j}(t+1)$ are running averages depending on the previous raw change signals $\hat{\delta} a_{i j}(t), \hat{\delta} b_{i j}(t)$

$$
\begin{aligned}
& \delta b_{i j}(t+1)=0.01 \delta b_{i j}(t)+0.99 \hat{\delta} b_{i j}(t) \\
& \delta a_{i j}(t+1)=0.01 \delta a_{i j}(t)+0.99 \hat{\delta} a_{i j}(t)
\end{aligned}
$$



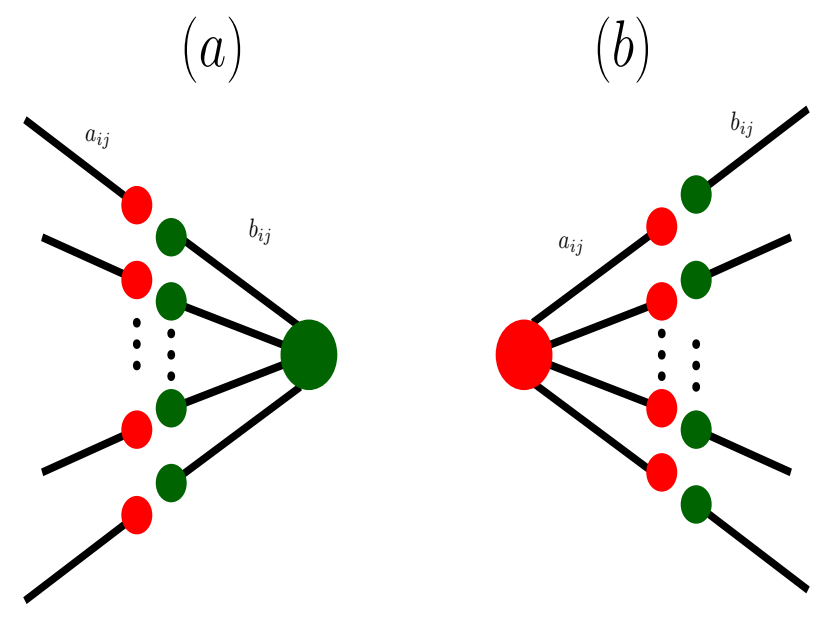

Fig 9. Illustration of synaptic competition induced by hetero-synaptic plasticity. a: Competition induced by post-synaptic hetero-synaptic plasticity. Excitatory pre-synaptic neurons i target a post-synaptic neuron $\mathrm{j}$. We assume that the resources required for increasing the post-synaptic components $b_{i j}$ of the weights $w_{i j}=a_{i j} b_{i j}$ are limited and therefore distributed in a competitive manner. That is, we assume hetero-synaptic plasticity where afferent synapses that receive large eligibility signals $\varepsilon_{i j}$ increase their efficacy while synapses that receive weaker (but for excitatory synapses always positive) signals will instead weaken. b: Pre-synaptically induced competition is assumed to follow the same principle. The signals for the changes of the pre-synaptic components $a_{i j}$, however, are assumed to depend on the realized amount of potentiation at the post-synaptic side, i.e. to take the post-synaptic hetero-synaptic competition into account. We found that this choice is more parameter tolerant and yields more robust memory than a symmetric version where pre- and post-synaptic hetero-synaptic plasticity are based on the same eligibility signal which, however, can also realize self-organized spike pattern detection (not shown).

Note that $\hat{r}_{j}(0)=0, \hat{\delta} a_{i j}(0)=0$, and $\hat{\delta} b_{i j}(0)=0$. Because synaptic strength cannot become arbitrary strong due to synapses' structure and other constraints, we cut weights changes that would carry those synapses out of the bounds $w_{\text {bound }}$. $w_{\text {bound }}$ is plus and minus one for excitatory and inhibitory synapses, respectively.

Besides, Dale's rule dictates that excitatory and inhibitory synapses cannot turn to each other; therefore, we assume synaptic weights become zero if weight changes would turn their kind during the learning. As a result, subtracting the mean value in equations 11 and 17 will not change a synapse's type.

To convert each afferent's time code input to rate code input, we first convolve each spike with a Gaussian distribution with a zero mean and a standard deviation of $20 \mathrm{~ms}$. The resulting function is then used as modulated firing rate of a Poisson point process. This results in an ensemble of spatio-temporal patterns based on the original pattern that has the statistics of Poisson processes, including failures and a Fano factor of 1 . These distorted patterns are then used for learning and testing.

\section{Acknowledgments}

This work was supported by DFG - grant (PA 569/5-1) 


\section{References}

1. Reinagel, P. \& Reid, R. C. Temporal coding of visual information in the thalamus. Journal of Neuroscience 20, 5392-5400 (2000).

2. Uzzell, V. \& Chichilnisky, E. Precision of spike trains in primate retinal ganglion cells. Journal of Neurophysiology (2004).

3. Ince, R. A., Panzeri, S. \& Kayser, C. Neural codes formed by small and temporally precise populations in auditory cortex. Journal of Neuroscience 33, 18277-18287 (2013).

4. Reich, D. S., Mechler, F. \& Victor, J. D. Independent and redundant information in nearby cortical neurons. Science 294, 2566-2568 (2001).

5. Johansson, R. S. \& Birznieks, I. First spikes in ensembles of human tactile afferents code complex spatial fingertip events. Nature neuroscience 7, 170-177 (2004).

6. Wehr, M. \& Laurent, G. Odour encoding by temporal sequences of firing in oscillating neural assemblies. Nature 384, 162-166 (1996).

7. Carr, C. \& Konishi, M. A circuit for detection of interaural time differences in the brain stem of the barn owl. Journal of Neuroscience 10, 3227-3246 (1990).

8. Decharms, R. C. \& Merzenich, M. M. Primary cortical representation of sounds by the coordination of action-potential timing. Nature 381, 610-613 (1996).

9. Gütig, R. \& Sompolinsky, H. The tempotron: a neuron that learns spike timing-based decisions. Nature neuroscience 9, 420-428 (2006).

10. Florian, R. The chronotron: a neuron that learns to fire temporally-precise spike patterns. Nature Precedings 1-1 (2010).

11. White, B. Principles of neurodynamics: Perceptrons and the theory of brain mechanisms (1963).

12. Rubin, R., Monasson, R. \& Sompolinsky, H. Theory of spike timing-based neural classifiers. Physical review letters 105, 218102 (2010).

13. Yu, Q., Tang, H., Tan, K. C. \& Li, H. Precise-spike-driven synaptic plasticity: Learning heteroassociation of spatiotemporal spike patterns. Plos one 8, e78318 (2013).

14. Albers, C., Westkott, M. \& Pawelzik, K. Perfect associative learning with spike-timing-dependent plasticity. In Proceedings of the 26th International Conference on Neural Information Processing Systems-Volume 2, 1709-1717 (2013).

15. Gütig, R. Spiking neurons can discover predictive features by aggregate-label learning. Science 351 (2016).

16. Turrigiano, G. G. The self-tuning neuron: synaptic scaling of excitatory synapses. Cell 135, 422-435 (2008).

17. Chistiakova, M., Bannon, N. M., Chen, J.-Y., Bazhenov, M. \& Volgushev, M. Homeostatic role of heterosynaptic plasticity: models and experiments. Frontiers in computational neuroscience $\mathbf{9}, 89$ (2015).

18. Okun, M. \& Lampl, I. Instantaneous correlation of excitation and inhibition during ongoing and sensory-evoked activities. Nature neuroscience 11, 535-537 (2008).

19. Isaacson, J. S. \& Scanziani, M. How inhibition shapes cortical activity. Neuron 72, 231-243 (2011). 
20. Denève, S. \& Machens, C. K. Efficient codes and balanced networks. Nature neuroscience 19, 375-382 (2016).

21. Parisi, G. I., Kemker, R., Part, J. L., Kanan, C. \& Wermter, S. Continual lifelong learning with neural networks: A review. Neural Networks 113, 54-71 (2019).

22. Tong, R., Chater, T. E., Emptage, N. J. \& Goda, Y. Heterosynaptic cross-talk of pre-and postsynaptic strengths along segments of dendrites. Cell Reports 34, 108693 (2021).

23. Mainen, Z. F. \& Sejnowski, T. J. Reliability of spike timing in neocortical neurons. Science 268, 1503-1506 (1995).

24. Vogels, T. P., Sprekeler, H., Zenke, F., Clopath, C. \& Gerstner, W. Inhibitory plasticity balances excitation and inhibition in sensory pathways and memory networks. Science 334, 1569-1573 (2011).

25. Sadeh, S. \& Clopath, C. Inhibitory stabilization and cortical computation. Nature Reviews Neuroscience 22, 21-37 (2021).

26. Litwin-Kumar, A. \& Doiron, B. Formation and maintenance of neuronal assemblies through synaptic plasticity. Nature communications 5, 1-12 (2014).

27. Haider, B., Duque, A., Hasenstaub, A. R. \& McCormick, D. A. Neocortical network activity in vivo is generated through a dynamic balance of excitation and inhibition. Journal of Neuroscience 26, 4535-4545 (2006).

28. Rasmussen, C. E. \& Willshaw, D. J. Presynaptic and postsynaptic competition in models for the development of neuromuscular connections. Biological cybernetics 68, 409-419 (1993).

29. Wang, H. \& Wagner, J. J. Priming-induced shift in synaptic plasticity in the rat hippocampus. Journal of neurophysiology 82, 2024-2028 (1999). 